ARTICLE

\title{
Giant barocaloric effects at low pressure in ferrielectric ammonium sulphate
}

P. Lloveras ${ }^{1}$, E. Stern-Taulats ${ }^{2}$, M. Barrio ${ }^{1}$, J.-LI. Tamarit ${ }^{1}$, S. Crossley ${ }^{3}$, W. Li ${ }^{3,4}$, V. Pomjakushin ${ }^{5}$, A. Planes ${ }^{2}$, LI. Mañosa ${ }^{2}$, N.D. Mathur ${ }^{3}$ \& X. Moya ${ }^{2,3}$

Caloric effects are currently under intense study due to the prospect of environment-friendly cooling applications. Most of the research is centred on large magnetocaloric effects and large electrocaloric effects, but the former require large magnetic fields that are challenging to generate economically and the latter require large electric fields that can only be applied without breakdown in thin samples. Here we use small changes in hydrostatic pressure to drive giant inverse barocaloric effects near the ferrielectric phase transition in ammonium sulphate. We find barocaloric effects and strengths that exceed those previously observed near magnetostructural phase transitions in magnetic materials. Our findings should therefore inspire the discovery of giant barocaloric effects in a wide range of unexplored ferroelectric materials, ultimately leading to barocaloric cooling devices.

\footnotetext{
${ }^{1}$ Departament de Física i Enginyeria Nuclear, ETSEIB, Universitat Politècnica de Catalunya, Diagonal 647, Barcelona, 08028 Catalonia, Spain. ${ }^{2}$ Facultat de Física, Departament d'Estructura i Constituents de la Matèria, Universitat de Barcelona, Martí i Franquès 1, Barcelona, 08028 Catalonia, Spain. ${ }^{3}$ Department of Materials Science, University of Cambridge, 27 Charles Babbage Road, Cambridge CB3 OFS, UK. ${ }^{4}$ School of Physics and Wuhan National High Magnetic Field Center, Huazhong University of Science and Technology, Wuhan 430074, China. ${ }^{5}$ Laboratory for Neutron Scattering and Imaging, Paul Scherrer Institut (PSI), CH-5232 Villigen PSI, Switzerland. Correspondence and requests for materials should be addressed to X.M. (email: xm212@cam.ac.uk).
} 
F oodstuffs, beverages, medicine, electronics and populated spaces all require cooling, but existing refrigeration and airconditioning units rely primarily on the compression and expansion of environmentally harmful fluids. Resurgent interest in solid materials that display magnetically, electrically and mechanically driven phase transitions near room temperature ${ }^{1-3}$ has provoked interest in the possibility of environment-friendly cooling applications, but these will only come to fruition if it is possible to develop or discover inexpensive materials that show large reversible thermal changes in response to fields that are small and easy to generate.

Mechanical stress is easy to generate, but large barocaloric (BC) effects driven by hydrostatic pressure near phase transitions have only been observed in a small number of relatively expensive magnetic materials, where changes of magnetization are accompanied by changes in crystal symmetry ${ }^{4,5}$ or volume alone $e^{6-8}$ (Table 1). (Large BC effects have also been observed in poly(methyl methacrylate) away from any transition ${ }^{9}$.) Here we demonstrate giant $\mathrm{BC}$ effects near the ferrielectric phase transition ${ }^{10-13}$ in a powder of ammonium sulphate (AS) $\left[\left(\mathrm{NH}_{4}\right)_{2} \mathrm{SO}_{4}\right]$, which is made from cheap abundant elements and enjoys widespread agricultural use as a fertilizer. We use calorimetry to identify pressure-driven isothermal entropy changes of $|\Delta S| \sim 60 \mathrm{~J} \mathrm{~K}^{-1} \mathrm{~kg}^{-1}$, which exceed the corresponding values that have been found for metallic alloys near first-order magnetic phase transitions $\left(\sim 10-25 \mathrm{~J} \mathrm{~K}^{-1} \mathrm{~kg}^{-1}\right.$; Table 1), and predicted for $\mathrm{PbTiO}_{3}$ and $\mathrm{BaTiO}_{3}$ near first-order ferroelectric phase transitions $s^{14,15}\left(\sim 3-4 \mathrm{JK}^{-1} \mathrm{~kg}^{-1}\right)$. These giant entropy changes are driven using small changes of hydrostatic pressure $|\Delta p|=\left|p-p_{\text {atm }}\right| \sim|p| \sim 0.1 \mathrm{GPa}$, yielding giant $\mathrm{BC}$ strengths ${ }^{1}|\Delta S| /|\Delta p|,|Q| /|\Delta p|$ and $|\Delta T| /|\Delta p|$ (Table 1) (where $Q$ is the heat, $T$ is the temperature and atmospheric pressure $p_{\text {atm }} \sim 0 \mathrm{GPa}$ ). Our giant BC effects may be understood via pressure-driven changes in ionic ordering, whereas the smaller $\mathrm{BC}$ effects in magnetic materials ${ }^{4-8}$ arise due to pressure-driven changes in the density of electronic states near the Fermi level.

\section{Results}

Ferrielectric phase transition in AS at atmospheric pressure. At room temperature, AS adopts a centrosymmetric orthorhombic structure (Pnam) with four formula units per unit cell comprising three ionic groups (Fig. 1a) that are understood to adopt a disordered configuration at any given instant ${ }^{16,17}$. On cooling, the material is generally considered to undergo a reversible orderdisorder phase transition to an orthorhombic polar structure $\left(P n a 2_{1}\right)$ that is ferrielectric ${ }^{10,11}$. Our heat flow $\mathrm{dQ} / \mathrm{d} T$ measurements confirm that this transition occurs in two steps ${ }^{10-}$ 13. First, the symmetry change arises from a non-isochoric firstorder transition at $T_{1} \sim 221 \mathrm{~K}$ associated with partial ionic ordering (Fig. 1b). Second, further ordering yields additional changes of volume in a continuous manner down to $\sim 160 \mathrm{~K}$ (Fig. 1b-d). (Figure 1d was obtained using temperature-dependent lattice parameters (Supplementary Fig. 1) calculated from X-ray diffraction patterns (Supplementary Fig. 2).) The first-order transition is weakly hysteretic and occurs at $T_{1} \sim 224 \mathrm{~K}$ on heating. Its start and finish temperatures on cooling are $T_{\mathrm{c} 1} \sim 223 \mathrm{~K}$ and $T_{\mathrm{c} 2} \sim 216 \mathrm{~K}$, respectively, and its start and finish temperatures on heating are $T_{\mathrm{h} 1} \sim 222 \mathrm{~K}$ and $T_{\mathrm{h} 2} \sim 229 \mathrm{~K}$, respectively.

Integration of $(\mathrm{d} Q / \mathrm{d} T) / T$ yields the corresponding entropy change $\Delta S(T)$ (Fig. 1c), with $\left|\Delta S_{\mathrm{f}}\right|=130 \pm 6 \mathrm{~J} \mathrm{~K}^{-1} \mathrm{~kg}^{-1}$ for the full transition. Integration of $\mathrm{d} Q / \mathrm{d} T$ across the full transition yields a corresponding heat of $\left|Q_{\mathrm{f}}\right|=29 \pm 2 \mathrm{~kJ} \mathrm{~kg}^{-1}$. These values are in good agreement with previous experimental values ${ }^{12,13}$ of $\left|\Delta S_{\mathrm{f}}\right| \sim 126-133 \mathrm{~J} \mathrm{~K}^{-1} \mathrm{~kg}^{-1}$ and $\left|Q_{\mathrm{f}}\right| \sim 28-30 \mathrm{~kJ} \mathrm{~kg}^{-1}$, and are consistent with the change of entropy $\left|\Delta S_{\mathrm{f}}\right|=3 R \ln 2=130 \mathrm{JK}^{-1} \mathrm{~kg}^{-1}$ expected $^{16}$ for an orderdisorder transition involving three ionic groups per formula unit $\left(R=8.314 \mathrm{~J} \mathrm{~K}^{-1} \mathrm{~mol}^{-1}\right)$. For the first-order transition alone, integration yields $\left|\Delta S_{1}\right|=65 \pm 4 \mathrm{~J} \mathrm{~K}^{-1} \mathrm{~kg}^{-1}$ and latent heat $\left|Q_{1}\right|=14.5 \pm 1.0 \mathrm{~kJ} \mathrm{~kg}^{-1}$. These values correspond to $\sim 50 \%$ of the aforementioned values for the full transition and closely match literature values ${ }^{13}$ of $\left|\Delta S_{1}\right|=61 \mathrm{JK}^{-1} \mathrm{~kg}^{-1}$ and $\left|Q_{1}\right|=13.6 \mathrm{~kJ} \mathrm{~kg}^{-1}$ for deuterated $\mathrm{AS}\left[\left(\mathrm{ND}_{4}\right)_{2} \mathrm{SO}_{4}\right]$, where no aspect of the transition is modified by the deuteration.

On heating through the ferrielectric transition, X-ray diffraction data confirm the expected changes in crystal structure ${ }^{10,11,13}$. The unit-cell volume $V$ decreases by $\sim 0.9 \%$ across the full transition $\left(\Delta V_{\mathrm{f}}=-4.4 \pm 0.2 \AA^{3}\right)$ and by $\sim 0.5 \%$ across the first-order transition alone $\left(\Delta V_{1}=-2.5 \pm 0.2 \AA^{3}\right)$ (Fig. 1d). Given that $\mathrm{BC}$ effects per unit mass $m$ due to pressure change $\Delta p=p_{2}-p_{1}$ may be expressed using the Maxwell relation $m^{-1}(\partial V / \partial T)_{p}=-(\partial S / \partial p)_{T}$ as ${ }^{1} \Delta S\left(p_{1} \rightarrow p_{2}\right)=-m^{-1} \int_{p_{1}}^{p_{2}}(\partial V / \partial T)_{p^{\prime}} \mathrm{d} p^{\prime}$, we anticipate inverse $\mathrm{BC}$ effects in the transition regime where $(\partial V / \partial T)_{p=0}<0$ and we anticipate conventional $\mathrm{BC}$ effects away from the transition regime where $(\partial V / \partial T)_{p=0}>0$.

Ferrielectric phase transition in AS under applied pressure. For the first-order transition, heat flow measurements $\mathrm{d} Q / \mathrm{d} T$ reveal a strong pressure-induced shift in $T_{1}$ (Fig. 2a,b), with $\mathrm{d} T_{1} / \mathrm{d} p=$ $-57 \pm 4 \mathrm{~K} \mathrm{GPa}^{-1}$ on heating and $\mathrm{d} T_{1} / \mathrm{d} p=-45 \pm 4 \mathrm{~K} \mathrm{GPa}^{-1}$ on cooling. A similar shift of $-45 \pm 6 \mathrm{KGPa}^{-1}$ on heating is obtained via the Clausius-Clapeyron equation $\mathrm{d} T_{1} / \mathrm{d} p=\Delta v_{1} / \Delta S_{1}$, using $\Delta S_{1}=65 \pm 4 \mathrm{JK}^{-1} \mathrm{~kg}^{-1}$ (Fig. 1c) and specific volume change $\Delta v_{1}=-(2.9 \pm 0.2) \times 10^{-6} \mathrm{~m}^{3} \mathrm{~kg}^{-1}$ (from Fig. 1d). These large values of $\mathrm{d} T_{1} / \mathrm{d} p$ are similar to those reported for single-crystal $\mathrm{AS}^{18,19}$ and magnetic alloys (Table 2), and indicate that the narrow first-order transition of width $T_{\mathrm{c} 1}-T_{\mathrm{c} 2} \sim T_{\mathrm{h} 2}-T_{\mathrm{h} 1} \sim 7 \mathrm{~K}$ may be fully driven in either direction using moderate values of $|\Delta p| \sim 0.15 \mathrm{GPa}$.

Table 1 | Giant BC effects at first-order phase transitions.

\begin{tabular}{|c|c|c|c|c|c|c|c|c|c|c|}
\hline Giant BC material & $\begin{array}{l}\mathbf{T} \\
\mathbf{K} \\
\end{array}$ & $\begin{array}{c}|\Delta S| \\
\mathrm{JK}^{-1} \mathbf{k g}^{-1}\end{array}$ & $\begin{array}{c}|\Delta T| \\
K\end{array}$ & $\begin{array}{c}|Q| \\
\mathbf{k J ~}^{\mathbf{k g}^{-1}}\end{array}$ & $\begin{array}{l}|\Delta p| \\
\mathbf{G P a}\end{array}$ & $\begin{array}{c}|\Delta S / \Delta p| \\
\mathrm{JK}^{-1} \mathbf{k g}^{-1} \mathbf{G P a}^{-1}\end{array}$ & $\begin{array}{l}|\Delta T / \Delta p| \\
\mathrm{KGPa}^{-1} \\
\end{array}$ & $\begin{array}{c}|Q / \Delta p| \\
\mathrm{kJ} \mathrm{kg}^{-1} \mathrm{GPa}^{-1} \\
\end{array}$ & $\begin{array}{c}\mathbf{R C} \\
\mathrm{Jkg}^{-1}\end{array}$ & Ref. \\
\hline $\mathrm{Ni}_{49.26} \mathrm{Mn}_{36.08} \mathrm{In}_{14.66}$ & 293 & 24 & {$[4.5]$} & 7.1 & 0.26 & 92.3 & 17.3 & 27.3 & 120 & 4 \\
\hline $\mathrm{Gd}_{5} \mathrm{Si}_{2} \mathrm{Ge}_{2}$ & 270 & 11 & 1.1 & 2.9 & 0.20 & 55 & 5.5 & 14.5 & 81 & 5 \\
\hline $\mathrm{Fe}_{49} \mathrm{Rh}_{51}$ & 308 & 12.5 & {$[8.1]$} & 3.8 & 0.11 & 114 & 74 & 34.5 & 105 & 7 \\
\hline $\mathrm{Mn}_{3} \mathrm{GaN}$ & 285 & 21.6 & [4.8] & 6.2 & 0.09 & 232 & 51.4 & 66.2 & 125 & 8 \\
\hline AS & 219 & 60 & {$[8]$} & 13.2 & 0.10 & 600 & 80 & 132 & 276 & This work \\
\hline
\end{tabular}

$B C$, barocaloric; $|\Delta p|$, hydrostatic pressure change; $|Q|$, isothermal heat; $R C$, refrigerant capacity; $|\Delta S|$, isothermal entropy change; $T$, starting temperature; $|\Delta T|$, adiabatic temperature change. $|\Delta S|,|\Delta T|$ and $|Q|$ arise at $T$, due to changes of $|\Delta p|$. The corresponding strengths $|\Delta S| /|\Delta p|,|\Delta T| /|\Delta p|$ and $|Q| /|\Delta p|$ were maximized by choosing the smallest values of $|\Delta p|$ compatible with maximizing $|\Delta S|$. Bold entries denote data derived from direct measurements. Italicised entries denote data derived from quasi-direct ${ }^{1}$ measurements. Bracketed entries denote data derived via $-c \Delta T \simeq T \Delta S=Q$ using zero-pressure specific heat capacity $c$. For all entries, $Q=T \Delta S$. 
a
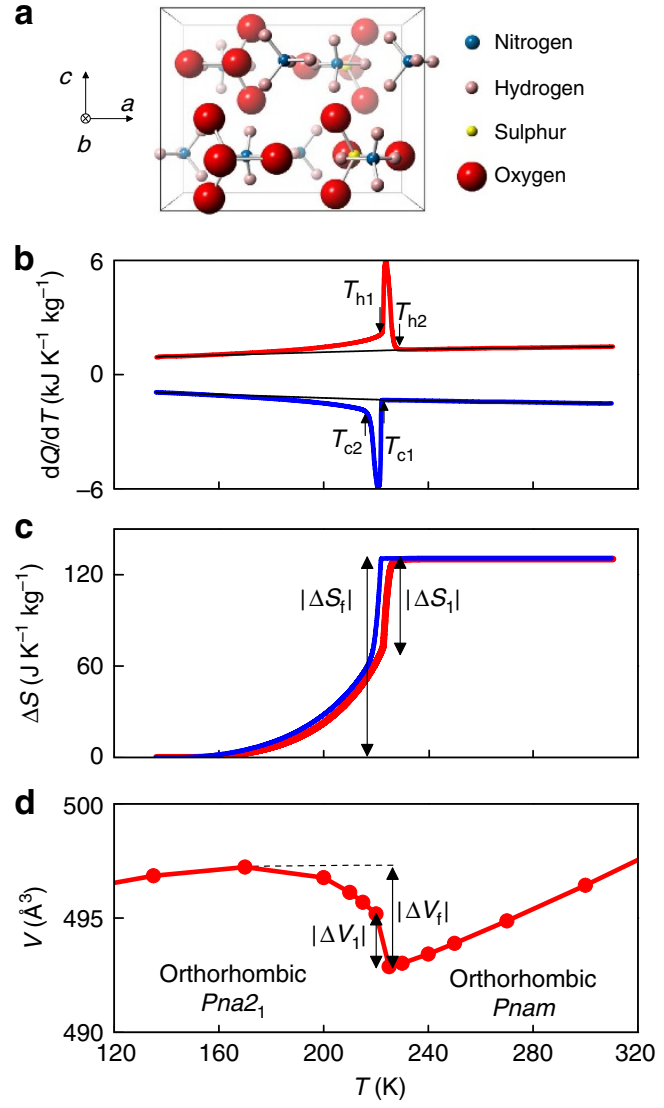

Figure 1 | Ferrielectric phase transition in AS at atmospheric pressure.

(a) Unit cell of the high-temperature orthorhombic phase (Pnam). (b) Heat flow $\mathrm{dQ} / \mathrm{d} T$ on cooling (blue) and heating (red) across the full transition. Baselines are black and $\mathrm{dQ} / \mathrm{d} T>0$ denotes endothermic processes.

(c) Resulting entropy change $\Delta S(T)$ with respect to the low-temperature phase, revealing entropy changes for the first-order transition $\left(\left|\Delta S_{1}\right|\right)$ and the entire transition $\left(\left|\Delta S_{f}\right|\right)$. (d) Unit-cell volume $V(T)$ on heating, revealing volume changes for the first-order transition $\left(\left|\Delta V_{1}\right|\right)$ and the entire transition $\left(\left|\Delta V_{f}\right|\right)$.

The discrepancy in values of $T_{1}(p)$ measured on heating and cooling (Fig. 2b) evidences a thermal hysteresis that is suppressed below the maximum value of $p \sim 0.3 \mathrm{GPa}$ for our calorimeter. At even higher pressures, neutron diffraction data for deuterated AS reveal that $\mathrm{d} T_{1} / \mathrm{d} p$ remains constant (open symbols, Fig. 2b), while $\left|\Delta V_{1}\right|$ falls (Fig. 2c), implying via the Clausius-Clapeyron equation a pressure-induced suppression of $\left|\Delta S_{1}\right|$. (Figure $2 \mathrm{c}$ was obtained using temperature-dependent lattice parameters (Supplementary Fig. 1) calculated from neutron diffraction patterns (Supplementary Fig. 3).) This suppression was confirmed (Fig. 2d) from finitepressure plots of $\left|\Delta S_{1}(T)\right|$ (Supplementary Fig. 4a,b) obtained from the calorimetric data of Fig. 2a, as described in Methods.

BC effects in AS. The fall in $\left|\Delta S_{1}(p)\right|$ arises because of additional changes in isothermal entropy $\Delta S_{+}(p)$ that are reversible, large and change sign across the first-order transition. Above $T_{1}(p)$, these additional entropy changes correspond to conventional BC effects associated with elastic heat, which arises at all temperatures, except while driving transitions. Near and below $T_{1}(p)$, these additional entropy changes correspond to inverse $\mathrm{BC}$ effects, because the continuous part of the full transition precludes elastic heat. The additional entropy changes would be challenging to detect via the calorimetry of Fig. 2, but they may be expressed $^{1}$ away from the first-order transition as $\Delta S\left(p_{1} \rightarrow p_{2}\right)=$ $-m^{-1} \int_{p_{1}}^{p_{2}}(\partial V / \partial T)_{p^{\prime}} \mathrm{d} p^{\prime}$, using the aforementioned Maxwell relation with $S_{+}$replacing $S$. From this formulation, we anticipate large values of $\Delta S_{+}$given an AS volumetric thermal expansion coefficient $V^{-1}(\partial V / \partial T)_{p}$ whose magnitude $\sim 10^{-4} \mathrm{~K}^{-1}$ (Supplementary Fig. 5a) exceeds the corresponding values ${ }^{6,20-24}$ of $\sim 10^{-7}-10^{-5} \mathrm{~K}^{-1}$ for the magnetic BC materials of Table 1 .

To confirm that the fall in $\left|\Delta S_{1}(p)\right|$ arises due to additional changes of entropy $\Delta S_{+}$away from $T_{1}(p)$, we evaluated $\Delta S_{+}(p)$ on applying pressure above $T_{1}(p)$ at $T_{+}=236 \mathrm{~K}$ by assuming $(\partial V / \partial T)_{p}$ to be independent of pressure such that $\Delta S_{+}$ $(p)=-\left[m^{-1}(\partial V / \partial T)_{p=0}\right] p$ (pressure-dependent data are unavailable due to inaccurate low-pressure control coupled with excessive neutron acquisition times). Choosing $T_{+}>T_{1}(p)$ is convenient, because it avoids the forbidden possibility of $T_{1}(p)$ falling to $T_{+}$at high pressure. Using the resulting values of $\Delta S_{+}(p)$ at $T_{+}=236 \mathrm{~K}$ (Supplementary Fig. 5b) to displace at this temperature the finitepressure plots of $\Delta S_{1}(T)$ (Supplementary Fig. 4a,b for heating and cooling, respectively), we have constructed finite-pressure plots of total entropy change $\Delta S(T, p)$ (Fig. 3a,b) specified with respect to the zero-pressure total entropy below the first-order transition at $208 \mathrm{~K}$. Whether the calorimetrically accessible value of $\Delta S_{1}(T)$ was measured on heating (for Fig. 3a) or cooling (for Fig. 3b), the resulting values of $\Delta S(208 \mathrm{~K}, p)$ match well with predictions of $\Delta S_{+}(p)$ that were obtained by setting $T_{+}$to $208 \mathrm{~K}$ (Supplementary Fig. $5 \mathrm{~b}$ ), thus providing quantitative confirmation that the fall in $\left|\Delta S_{1}(p)\right|$ arises due to the sign change in $\mathrm{BC}$ effects across the firstorder transition.

Our plot of $\Delta S(T, p)$ for data obtained on heating (Fig. 3a) permits us to establish isothermal $\mathrm{BC}$ effects on applying pressure (Fig. 3c), as heating and high pressure both tend to favour the high-temperature, high-pressure centrosymmetric phase. Similarly, our plot of $\Delta S(T, p)$ for data obtained on cooling (Fig. 3b) permits us to establish isothermal BC effects on decreasing pressure (Fig. 3c), as cooling and low pressure both tend to favour the low-temperature ferrielectric phase. Near and above the value of $T_{\mathrm{cl}}(p=0)$ indicated, discrepancies in isothermal entropy change on applying and removing pressure evidence irreversibility. By contrast, reversible BC effects are apparent a few degrees below $T_{\mathrm{c} 1}(p=0)$ and at all lower temperatures studied, consistent with no significant thermal hysteresis in the first-order transition (Fig. 2b). The largest reversible isothermal entropy change $|\Delta S| \sim 60 \pm 5 \mathrm{~J} \mathrm{~K}^{-1} \mathrm{~kg}^{-1}$ arises at $\sim 219 \mathrm{~K}$ and exceeds the giant $\mathrm{BC}$ effects reported for magnetic alloys (Table 1). The sharpness of the transition in $\Delta S(T)$ (Fig. 3a,b) permits this large entropy change to be achieved with a low value of $|\Delta p|=0.1 \mathrm{GPa}$ (Fig. 3c), yielding giant $\mathrm{BC}$ strengths ${ }^{1}|\Delta S| /|\Delta p|$ and $|Q| /|\Delta p|$ (Table 1). Larger pressures extend reversible BC effects to lower temperatures, causing the large refrigerant capacity ${ }^{25}$ $\mathrm{RC}=|\Delta S| \times(\mathrm{FWHM}$ of $\Delta S(T))$ (Table 1) to increase (Fig. 4) despite the small reduction in $\left|\Delta S_{1}(p)\right|$ (Fig. 2d) and therefore $|\Delta S(p)|$. For any given value of applied pressure, AS outperforms all of the magnetic alloys so well that comparable $\mathrm{RC}$ values would require much larger changes of pressure (Fig. 4).

Our largest value of $|\Delta S| \sim 60 \pm 5 \mathrm{~J} \mathrm{~K}^{-1} \mathrm{~kg}^{-1}$, arising due to $|\Delta p|=0.1 \mathrm{GPa}$ at $\sim 219 \mathrm{~K}$, corresponds to an adiabatic temperature change $|\Delta T|=(T / c)|\Delta S| \sim 8 \pm 1 \mathrm{~K}$, using a specific heat capacity $c=1,700 \pm 80 \mathrm{~J} \mathrm{~K}^{-1} \mathrm{~kg}^{-1}$ (Supplementary Fig. 6) that is assumed to be independent of pressure as usual ${ }^{4-8}$. The resulting value of $|\Delta T| /|\Delta p|$ is seen to be the largest observed for giant BC materials (Table 1).

\section{Discussion}

Our observation of giant reversible BC effects in ferrielectric salts made from inexpensive abundant elements should inspire the study of BC effects in similar materials, most immediately bulk 

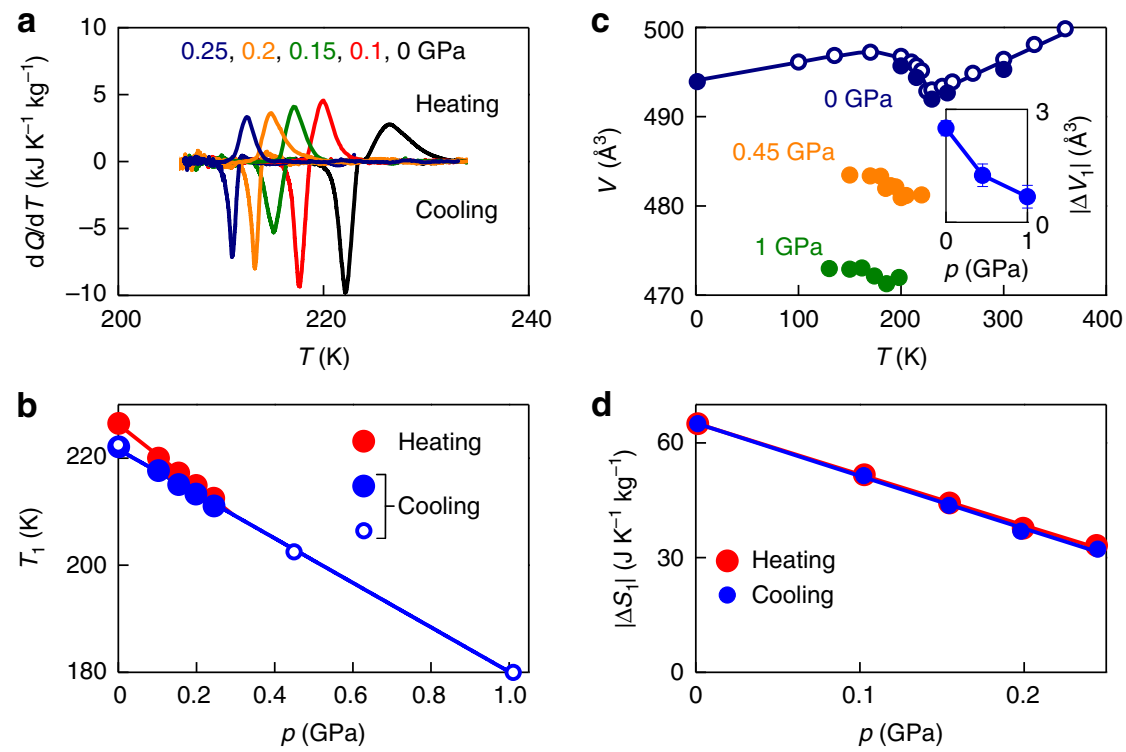

Figure 2 | Ferrielectric phase transition in AS under applied pressure. (a) Heat flow $d Q / d T$ on cooling and heating across the transition for different values of increasing pressure $p$, after baseline subtraction. (b) Transition temperature $T_{1}(p)$ for the first-order transition, obtained below $0.3 \mathrm{GPa}$ from the calorimetric data of $\mathbf{a}$ (closed symbols) and below $\sim 1.0 \mathrm{GPa}$ from neutron diffraction of deuterated AS (open symbols). (c) Unit-cell volume $V(T)$ obtained on cooling at selected pressures from neutron diffraction of deuterated AS (closed symbols), with inset showing $\left|\Delta V_{1}(p)\right|$. The $X$-ray diffraction data of Fig. 1d are included to demonstrate consistency (open symbols). (d) Entropy change $\left|\Delta S_{1}(p)\right|$ for the first-order transition, obtained from the calorimetric data of $\mathbf{a}$. Lines in $\mathbf{b}$ and $\mathbf{d}$ represent linear fits.

\section{Table 2 | Properties of first-order phase transitions in giant BC materials.}

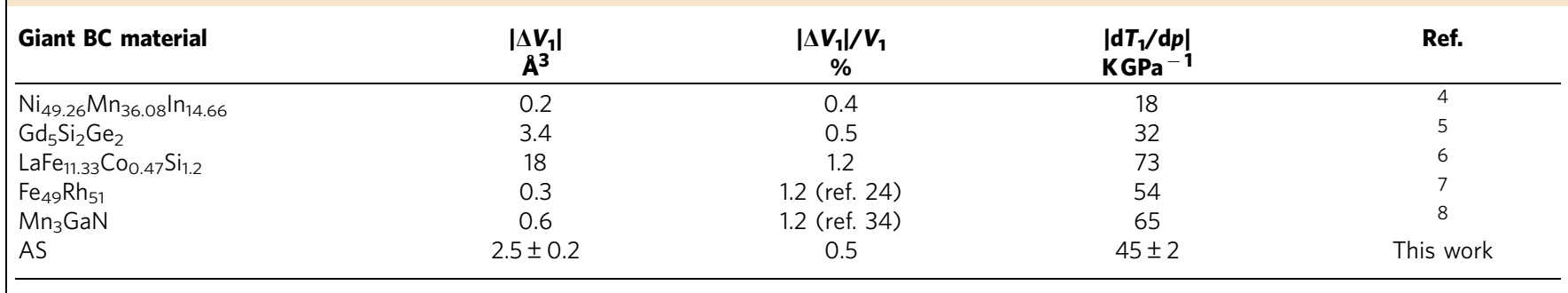

$\mathrm{AS}$, ammonium sulphate; $\mathrm{BC}$, barocaloric; $\left|\mathrm{d} T_{1} / \mathrm{d} p\right|$, pressure-driven shift in transition temperature; $\left|\Delta V_{1}\right|$, unit-cell volume change; $\left|\Delta V_{1}\right| / V_{1}$, relative unit-cell volume change.

For AS, we give the shift obtained over a wide pressure range on cooling.
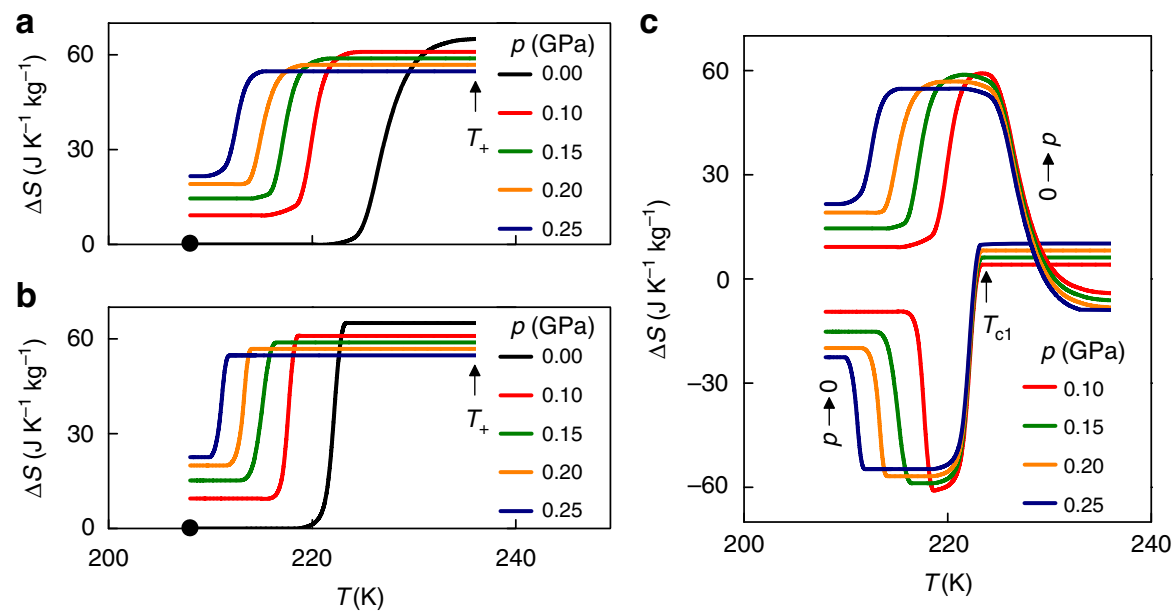

Figure 3 | Giant inverse BC effects in AS. (a,b) Entropy change $\Delta S(T, p)$ with respect to $S(T=208 \mathrm{~K}, p=0)$ (black dot), deduced using additional entropy change $\Delta S_{+}(p)$ at $T_{+}=236 \mathrm{~K}$, to offset the pressure-dependent entropy change $\Delta S_{1}(T)$ that arises on (a) heating and (b) cooling through the first-order transition. (c) Isothermal entropy change $\Delta S$ for increasing pressure $(0 \rightarrow p)$ as deduced from $\mathbf{a}$ and for decreasing pressure $(p \rightarrow 0)$ as deduced from $\mathbf{b}$. Reversibility is apparent up to a few degrees below $T_{\mathrm{c} 1}(p=0) \sim 223.5 \mathrm{~K}$. 


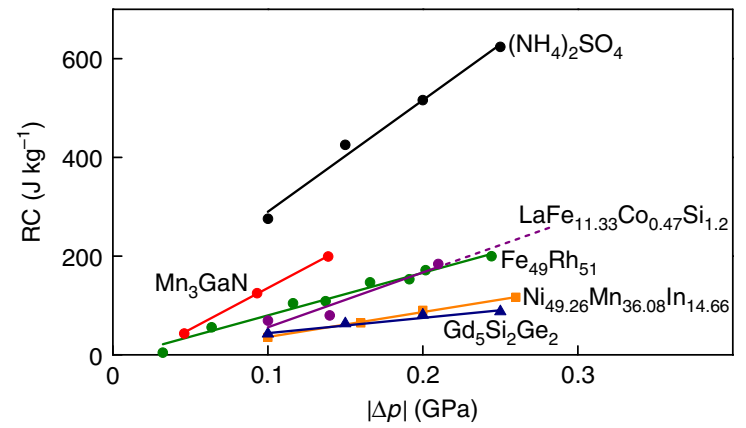

Figure 4 | Refrigerant capacity RC for giant BC materials. For the materials in Table 1, we compare values of $R C=|\Delta S| \times(F W H M$ of $\Delta S(T))$ for selected pressure changes of magnitude $|\Delta p|=\left|p-p_{\text {atm }}\right| \sim|p|$, using $\Delta S(T)$ for $B C$ cooling, and constraining $T$ to ensure reversibility (except for $\mathrm{LaFe}_{11.33} \mathrm{Co}_{0.47} \mathrm{Si}_{1.2}$, where only $\mathrm{BC}$ cooling data are available, and $\mathrm{Ni}_{49.26} \mathrm{Mn}_{36.08} \mathrm{In}_{14.66}$, where only $\mathrm{BC}$ heating data are available). Solid lines represent linear fits.

ferroelectrics that display large thermally driven entropy changes associated with displacive and order-disorder phase transitions. In future, it would be attractive to increase transition temperatures by chemical substitution ${ }^{26,27}$ or using an electric field ${ }^{28}$. It would also be attractive to perform direct thermal measurements in the vicinity of room temperature, to confirm the large BC effects predicted using the Maxwell relation (Supplementary Fig. 5b), which are reversible over a wide range of temperatures.

Our findings should stimulate the development of cooling devices based on BC materials, whose energy efficiency ${ }^{29,30}$ is good with respect to magnetocaloric, electrocaloric and elastocaloric materials ${ }^{3}$. Unlike elastocaloric materials driven by uniaxial stress, there are no losses or mechanical breakdown associated with plastic deformation. Unlike magnetocaloric materials, there is no need to generate large magnetic fields at great expense. Unlike electrocaloric materials, there is no need to fabricate multilayer devices to exploit giant effects in films ${ }^{31}$. Moreover, the phase transitions giving rise to large $\mathrm{BC}$ effects can be driven over a wide range of operating temperatures, unlike both magnetocaloric and electrocaloric materials.

\section{Methods}

Samples. Powders of AS ( $\geq 99.0 \%)$ and deuterated AS ( $\geq 99.0 \%)$ were purchased from Sigma-Aldrich. The typical grain size was $<100 \mu \mathrm{m}$. AS was used for calorimetry and X-ray diffraction. Deuterated AS was used for neutron diffraction to reduce incoherent scattering.

Calorimetry at atmospheric pressure. Measurements of heat flow $\mathrm{d} Q / \mathrm{d} T$ were performed at atmospheric pressure using a commercial TA Q2000 differential scanning calorimeter at $10 \mathrm{~K} \mathrm{~min}^{-1}$. Heat $\left|Q_{\mathrm{f}}\right|=\left|\int_{T_{\mathrm{a}}}^{T_{\mathrm{b}}}\left(\mathrm{d} Q / \mathrm{d} T^{\prime}\right) \mathrm{d} T^{\prime}\right|$ and entropy change $\left|\Delta S_{\mathrm{f}}\right|=\left|\int_{T_{\mathrm{b}}}^{T_{\mathrm{b}}}\left(\mathrm{d} Q / \mathrm{d} T^{\prime}\right) / T^{\prime} \mathrm{d} T^{\prime}\right|$ across the full transition were obtained after subtracting baseline backgrounds ${ }^{32}$, with $T_{\mathrm{a}}$ chosen above (below) the transition on cooling (heating) and $T_{\mathrm{b}}$ chosen below (above) the transition on cooling (heating). The entropy change on partially driving the transition by heating to temperature $T$ is $\Delta S(T)=\int_{T_{\mathrm{a}}}^{T}\left(\mathrm{dQ} / \mathrm{d} T^{\prime}\right) / T^{\prime} \mathrm{d} T^{\prime}$. The entropy change on partially driving the transition by cooling to temperature $T$ is $\Delta S(T)=\left|\Delta S_{\mathrm{f}}\right|-\int_{T}^{T}\left(\mathrm{~d} Q / \mathrm{d} T^{\prime}\right) / T^{\prime} \mathrm{d} T^{\prime}$.

Zero-field heat capacity data were obtained using the TA Q2000 on cooling in the modulated differential scanning calorimetry mode, with the constant temperature method ${ }^{33}$. The temperature step was $1 \mathrm{~K}$, the temperature modulation was $0.5 \mathrm{~K}$ and the period was $60 \mathrm{~s}$.

Calorimetry under applied pressure. Measurements of heat flow $\mathrm{d} Q / \mathrm{d} T$ at constant hydrostatic pressure were performed at $\pm 1-2 \mathrm{Kmin}^{-1}$, using a differential thermal analyser constructed in-house, with chromel-alumel thermocouples, a $\mathrm{Cu}-\mathrm{Be}$ Bridgman pressure cell operating up to $0.3 \mathrm{GPa}$ and a circulating thermal bath (Lauda Proline RP 1290, 183-473 K). AS was mixed with an inert perfluorinated liquid and hermetically encapsulated by Sn. DW-Therm (Huber Kältemaschinenbau $\mathrm{GmbH}$ ) was used as pressure-transmitting medium.
For more details, see refs 4-7. Absolute measurements of temperature in the differential thermal analyser and differential scanning calorimeter differ by $\sim 1 \mathrm{~K}$.

X-ray diffraction. High-resolution X-ray diffraction was performed in transmission using $\mathrm{Cu} K \alpha_{1}=1.5406 \AA$ radiation in an INEL diffractometer, with a curved position-sensitive detector (CPS120), a $0.5-\mathrm{mm}$ diameter Lindemann capillary and a 700 series Oxford Cryostream Cooler.

Neutron diffraction. High-resolution neutron diffraction was performed at the Paul Scherrer Institute, using the high-resolution powder diffractometer for thermal neutrons. Deuterated AS was mixed with $\mathrm{NaCl}$ powder to determine the applied pressure, and the mixture was encapsulated in a $\mathrm{Pb}$ clamp cell operating up to $\sim 1 \mathrm{GPa}$. Temperature was varied using a cryostat operating in $1.4-320 \mathrm{~K}$. The neutron wavelength was set to $1.88570 \AA$. Lattice parameters were determined by pattern matching using FullProf software.

Data availability. All relevant data are presented via this publication and Supplementary Information.

\section{References}

1. Moya, X., Kar-Narayan, S. \& Mathur, N. D. Caloric materials near ferroic phase transitions. Nat. Mater. 13, 439-450 (2014).

2. Mañosa, Ll., Planes, A. \& Acet, M. Advanced materials for solid-state refrigeration. J. Mater. Chem. A 1, 4925-4936 (2013).

3. Crossley, S., Mathur, N. D. \& Moya, X. New developments in caloric materials for cooling applications. AIP Advances 5, 067153 (2015).

4. Mañosa, Ll. et al. Giant solid-state barocaloric effect in the Ni-Mn-In magnetic shape-memory alloy. Nat. Mater. 9, 478-481 (2010).

5. Yuce, S. et al. Barocaloric effect in the magnetocaloric prototype $\mathrm{Gd}_{5} \mathrm{Si}_{2} \mathrm{Ge}_{2}$ Appl. Phys. Lett. 101, 071906 (2012).

6. Mañosa, Ll. et al. Inverse barocaloric effect in the giant magnetocaloric La-FeSi-Co compound. Nat. Commun. 2, 595 (2011).

7. Stern-Taulats, E. et al. Barocaloric and magnetocaloric effects in $\mathrm{Fe}_{49} \mathrm{Rh}_{51}$. Phys Rev. B 89, 214105 (2014).

8. Matsunami, D., Fujita, A., Takenaka, K. \& Kano, M. Giant barocaloric effect enhanced by the frustration of the antiferromagnetic phase in $\mathrm{Mn}_{3} \mathrm{GaN}$. Nat. Mater. 14, 73-78 (2015).

9. Rodriguez, E. L. \& Filisko, F. E. Thermoelastic temperature changes in poly(methyl methacrylate) at high hydrostatic pressure: experimental. J. Appl. Phys. 53, 6536-6540 (1982).

10. Unruh, H. G. \& Rüdiger, U. The ferroelectric transition of $\left(\mathrm{NH}_{4}\right)_{2} \mathrm{SO}_{4}$. J. Phys. Colloq. 33, C2-77-C2-78 (1972).

11. Sawada, A., Ohya, S., Ishibashi, Y. \& Takagi, Y. Ferroelectric phase transition in $\left(\mathrm{NH}_{4}\right)_{2} \mathrm{SO}_{4}-\mathrm{K}_{2} \mathrm{SO}_{4}$ mixed crystals. J. Phys. Soc. Jpn 38, 1408-1414 (1975).

12. Shomate, C. H. Specific heats at low temperatures of $\left(\mathrm{NH}_{4}\right)_{2} \mathrm{SO}_{4}, \mathrm{NH}_{4} \mathrm{Al}\left(\mathrm{SO}_{4}\right)_{2}$ and $\mathrm{NH}_{4} \mathrm{Al}\left(\mathrm{SO}_{4}\right)_{2} \cdot 12 \mathrm{H}_{2}$ O. J. Am. Chem. Soc. 67, 1096-1098 (1945).

13. Hoshino, S., Vedam, K., Okaya, V. \& Pepinsky, R. Dielectric and thermal study of $\left(\mathrm{NH}_{4}\right)_{2} \mathrm{SO}_{4}$ and $\left(\mathrm{NH}_{4}\right)_{2} \mathrm{BeF}_{4}$ transitions. Phys. Rev. 112, 405-412 (1958).

14. Mikhaleva, E. A. et al. Caloric characteristics of $\mathrm{PbTiO}_{3}$ in the temperature range of the ferroelectric phase transition. Phys. Solid State 54, 1832-1840 (2012).

15. Liu, Y. et al. Giant room-temperature barocaloric effect and pressure-mediated electrocaloric effect in $\mathrm{BaTiO}_{3}$ single crystal. Appl. Phys. Lett. 104, 162904 (2014).

16. O'Reilly, D. \& Tsang, T. Deuteron magnetic resonance and proton relaxation times in ferroelectric ammonium sulfate. J. Chem. Phys. 46, 1291-1300 (1967).

17. Dahlborg, U., Larsson, K. E. \& Pirkmajer, E. Rotational motions in solids. The ferroelectric transition in $\left(\mathrm{NH}_{4}\right)_{2} \mathrm{SO}_{4}$. Physica 49, 1-25 (1970).

18. Tsunekawa, S., Ishibashi, Y. \& Takagi, Y. Effect of the hydrostatic pressure on the transition temperature in $\left(\mathrm{NH}_{4}\right)_{2} \mathrm{SO}_{4}$. J. Phys. Soc. Jpn 33, 862 (1972).

19. Gaba, V. M. Baric shift of phase transition in ammonium sulfate crystals. Acta Phys. Pol. A 117, 126-128 (2010).

20. Aksoy, S. et al. Magnetization easy axis in martensitic Heusler alloys estimated by strain measurements under magnetic field. Appl. Phys. Lett. 91, 251915 (2007).

21. Li, B. et al. Magnetostructural coupling and magnetocaloric effect in Ni-Mn-In. Appl. Phys. Lett. 95, 172506 (2009).

22. Takenaka, K. \& Takagi, H. Giant negative thermal expansion in Ge-doped anti-perovskite manganese nitrides. Appl. Phys. Lett. 87, 261902 (2005).

23. Magen, C. et al. Hydrostatic pressure control of the magnetostructural phase transition in $\mathrm{Gd}_{5} \mathrm{Si}_{2} \mathrm{Ge}_{2}$ single crystals. Phys. Rev. B 72, 024416 (2005).

24. Annaorazov, M. P., Nikitin, S. A., Tyurin, A. L. \& Asatryan, K. A. Anomalously high entropy change in FeRh alloy. J. Appl. Phys. 79, 1689-1695 (1996).

25. Gschneidner, Jr K. A. \& Pecharsky, V. K. Magnetocaloric materials. Annu. Rev. Mater. Sci. 30, 387-429 (2000).

26. Mróz, B., Tuszynski, J. A., Kiefte, H. \& Clouter, M. J. On the ferroelastic phase transition of $\mathrm{LiNH}_{4} \mathrm{SO}_{4}$ : a Brillouin scattering study and theoretical modelling. J. Phys. Condens. Matter 1, 783-796 (1989). 
27. Ohi, K., Osaka, J. \& Uno, H. Ferroelectric phase transition in $\mathrm{Rb}_{2} \mathrm{SO}_{4}$ $\left(\mathrm{NH}_{4}\right)_{2} \mathrm{SO}_{4}$ and $\mathrm{Cs}_{2} \mathrm{SO}_{4}-\left(\mathrm{NH}_{4}\right)_{2} \mathrm{SO}_{4}$ mixed crystals. J. Phys. Soc. Jpn 44, 529-536 (1978).

28. Kamiyoshi, K.-I. Effect of electric field on the phase transition of ammonium sulfate. J. Chem. Phys. 26, 218-219 (1957).

29. Defay, E., Crossley, S., Kar-Narayan, S., Moya, X. \& Mathur, N. D. The electrocaloric efficiency of ceramic and polymer films. Adv. Mater. 25, 3337-3342 (2013).

30. Moya, X., Defay, E., Heine, V. \& Mathur, N. D. Too cool to work. Nat. Phys. 11, 202-205 (2015).

31. Kar-Narayan, S. \& Mathur, N. D. Predicted cooling powers for multilayer capacitors based on various electrocaloric and electrode materials. Appl. Phys. Lett. 95, 242903 (2009).

32. Ortín, J. \& Planes, A. Thermodynamic analysis of thermal measurements in thermoelastic martensitic transformations. Acta Metall. 36, 1873-1889 (1988).

33. Boller, A., Jin, Y. \& Wunderlich, B. Heat capacity measurements by modulated DSC at constant temperature. J. Therm. Anal. 42, 307-330 (1994).

34. Lukashev, P., Sabirianov, R. F. \& Belaschenko, K. Theory of the piezomagnetic effect in Mn-based antiperovskites. Phys. Rev. B 78, 184414 (2008).

\section{Acknowledgements}

This work was supported by the UK EPSRC grant EP/M003752/1, by CICyT (Spain) project numbers MAT2013-40590-P and FIS2011-24439, and by DGU (Catalonia) project 2014SGR00581. We thank D. Sheptyakov for assistance with high-pressure neutron diffraction. P.Ll. acknowledges support from SUR (DEC, Catalonia). E.S.-T. acknowledges support from AGAUR. W.L. acknowledges support from the Key Laboratory of Cryogenics (TIPC, CAS) and the NSFC. X.M. is grateful for support from the Spanish MEC Ramón y Cajal programme and the Royal Society.

\section{Author contributions}

X.M. conceived the study and led the project. X.M., Ll.M., A.P., P.Ll., M.B. and J.-Ll.T. planned the experiments. P.Ll., E.S.-T. and X.M. performed the calorimetric measurements at atmospheric pressure. P.Ll. and M.B. performed the calorimetric measurements under pressure. M.B. performed the X-ray diffraction measurements. P.Ll. and X.M. performed the neutron diffraction measurements, with support from V.P. X.M. wrote the manuscript with N.D.M. using substantive feedback from P.Ll., M.B., J.Ll.T., A.P. and Ll.M. E.S.-T., S.C., W.L. and V.P. also contributed to the preparation of the manuscript.

\section{Additional information}

Supplementary Information accompanies this paper at http://www.nature.com/ naturecommunications

Competing financial interests: The authors declare no competing financial interests.

Reprints and permission information is available online at http://npg.nature.com/ reprintsandpermissions/

How to cite this article: Lloveras, P. et al. Giant barocaloric effects at low pressure in ferrielectric ammonium sulphate. Nat. Commun. 6:8801 doi: 10.1038/ncomms9801 (2015).

(c) (i) This work is licensed under a Creative Commons Attribution 4.0 International License. The images or other third party material in this article are included in the article's Creative Commons license, unless indicated otherwise in the credit line; if the material is not included under the Creative Commons license, users will need to obtain permission from the license holder to reproduce the material. To view a copy of this license, visit http://creativecommons.org/licenses/by/4.0/ 